\title{
Platelet-derived Growth Factor Inhibits Bone Regeneration Induced by Osteogenin, a Bone Morphogenetic Protein, in Rat Craniotomy Defects
}

\author{
Leslie J. Marden, ${ }^{*}$ Robert S. P. Fan, * Glenn F. Pierce, ${ }^{3}$ A. Hari Reddi," and Jeffrey O. Hollinger * \\ * Department of Physiology, U.S. Army Institute of Dental Research, Walter Reed Army Medical Center, Washington, DC 20307-5300; \\ ${ }^{\S}$ Department of Experimental Pathology, Amgen, Inc., Thousand Oaks, California 91320; and "Johns Hopkins University School of \\ Medicine, Baltimore, Maryland 21205-2106
}

\begin{abstract}
Platelet-derived growth factor (PDGF) is a potent moderator of soft tissue repair through induction of the inflammatory phase of repair and subsequent enhanced collagen deposition. We examined the effect of recombinant BB homodimer PDGF (rPDGF-BB) applied to rat craniotomy defects, treated with and without bovine osteogenin (OG), to see if bone regeneration would be stimulated. Implants containing $0,20,60$, or 200 $\mu \mathrm{g}$ rPDGF-BB, reconstituted with insoluble rat collagenous bone matrix containing 0,30 , or $150 \mu \mathrm{g} O G$, were placed into 8-mm craniotomies. After 11 d, 21 of the 144 rats presented subcutaneous masses superior to the defect sites. The masses, comprised of serosanguinous fluid encapsulated by fibrous connective tissue, were larger and occurred more frequently in rats treated with $200 \mu \mathrm{g}$ rPDGF-BB, and were absent in rats not treated with rPDGF-BB. The masses underwent resorption within $28 \mathrm{~d}$ after surgery. OG (2-256 $\mu \mathrm{g})$ caused a dose-dependent increase in radiopacity and a marked regeneration of calcified tissue in a dose-dependent fashion within defect sites. However, OG-induced bone regeneration was inhibited $17-53 \%$ in the presence of $\mathrm{rPDGF}-\mathrm{BB}$. These results suggest that rPDGF-BB inhibited OG-induced bone regeneration and stimulated a soft tissue repair wound phenotype and response.( $J$. Clin. Invest. 1993. 92:2897-2905.) Key words: wound repair • critical-size defect $\bullet$ calvaria $\bullet$ dose dependent
\end{abstract}

\section{Introduction}

There is growing evidence that growth factors such as PDGF contribute to bone wound repair by attracting and stimulating proliferation of wound-healing cells and bone progenitor cells (1). Studies in vitro indicate that PDGF is chemotactic for neutrophils, monocytes, and fibroblasts (2-4), and stimulates the proliferation and activity of fetal $(5,6)$ and mature bone cells $(7,8)$. In vivo, PDGF stimulates granulation tissue formation in PDGF/collagen-treated chambers implanted subcutaneously $(9,10)$. Furthermore, PDGF stimulates healing of skin incisions and excisions by increasing inflammatory cell infiltration, provisional matrix formation, collagen deposition, and neovascularization (11-17).

Address correspondence to Jeffrey O. Hollinger, DDS, PhD, School of Medicine, Division of Plastic and Reconstructive Surgery, L352A, Oregon Health Sciences University, 3181 Southwest Sam Jackson Park Road, Portland, OR 97201-3098. 1993.

Received for publication 4 March 1993 and in revised form 9 July

The Journal of Clinical Investigation, Inc.

Volume 92, December 1993, 2897-2905
The initial stages of bone wound healing are similar to those observed in soft tissue. Blood from the marrow cavity and adjacent soft tissues fills the wound and a clot forms, followed by inflammation and granulation tissue formation. Progenitor cells are drawn to the wound, proliferate, and differentiate to cartilage- and bone-forming cells. The wound is revascularized, matrix is elaborated and mineralized, and hematopoiesis is restored. Many of the initial processes of bone wound repair, such as infiltration and proliferation of precursor cells, could be stimulated by PDGF, which might accelerate the entire bone repair process.

Few studies have tested the effect of PDGF on bone regeneration in vivo. In two studies, PDGF enhanced demineralized bone matrix-induced ectopic cartilage and bone formation $(18,19)$. However, PDGF had no effect when injected daily onto the periosteum of parietal bones of newborn rats, though similar treatment with transforming growth factor- $\beta$ doubled the thickness of calvariae at injection sites after $12 \mathrm{~d}(20,21)$.

Therapeutic concentrations of soluble proteins are difficult to maintain within wound beds, as factors may be washed away or diluted by extracellular fluids. Periodic addition of factors requires invasive procedures, such as injection (21-23) or infusion $(24,25)$, which are clinically impractical and may exacerbate the wound. Insoluble collagenous bone matrix (ICBM) ${ }^{1}$ has been used successfully as a carrier for bone-inductive proteins in vivo (26-28). The affinity of PDGF towards negatively charged extracellular matrix proteins present in ICBM (29) made it an ideal candidate for a biological carrier. However, as the activity of PDGF bound to matrix proteins is uncertain, this affinity had to be considered in estimating effective doses of PDGF.

Our laboratory has developed experimental intraosseous wound models, critical size defects (CSDs), for evaluating potential bone regenerative materials. If left untreated, CSDs will not heal by bone formation within the lifetime of the animal (30). The $8-\mathrm{mm}$ rat craniotomy is a CSD that heals not by bone formation but rather by fibrous union (31). Treatment of these CSDs with osteogenin (OG), a bone-inductive protein prepared from demineralized bone matrix, and ICBM results in complete bone regeneration within $28 \mathrm{~d}$ (28). OG induces the differentiation of pluripotential cells into osteoblasts, and stimulates the activity of alkaline phosphatase, but is not mitogenic for bone cells $(32,33)$. On the basis of available data, we hypothesized that treatment of rat craniotomy CSDs with PDGF would stimulate repair processes complementary to

1. Abbreviations used in this paper: BMP, bone morphogenetic protein; CSD, critical size defects; ICBM, insoluble collagenous bone matrix; OG, osteogenin. 
those induced by OG, and thereby further improve bone regeneration within the defect. Unexpectedly, PDGF antagonized the OG effect and appeared to direct repair toward a soft tissue phenotype.

\section{Methods}

Preparation of experimental materials. OG was partially purified from bovine demineralized bone matrix by hydroxyapatite, heparin sepharose, and size-exclusion liquid chromatography as previously described (34). This preparation contains bone morphogenetic protein (BMP)-2 and BMP-7 as well as OG (BMP-3), but no detectable PDGF, basic fibroblast growth factor, or transforming growth factor- $\beta$ (our unpublished observations). The preparation was dissolved in $5 \mathrm{mM} \mathrm{HCl}$ before use. ICBM was prepared from rat long bone demineralized with $0.5 \mathrm{~N} \mathrm{HCl}$ and extracted with $4 \mathrm{M}$ guanidine, $50 \mathrm{mM}$ Tris- $\mathrm{HCl}, \mathrm{pH} 7.4$, which rendered the material inactive as measured by heterotopic bioassay in rats (28). The insoluble residue was washed thoroughly with deionized water and lyophilized. Human recombinant (r)PDGF-BB was expressed in Escherichia coli and purified by standard chromatographic techniques (17). The protein was homogenous and contained no detectable endotoxin.

Implant preparation. $\mathrm{OG}$ and $0.75 \mathrm{mg}$ chondroitin sulfate (ICN ImmunoBiologicals, Elkheart, IN), in deionized water, were added to $25 \mathrm{mg}$ ICBM in 2-ml microcentrifuge tubes (8-mm internal diameter). The proteins were precipitated by the addition of dry ice-chilled absolute ethanol (35). The pellets obtained by centrifugation at $12,000 \mathrm{rpm}$ for 15 min were washed twice with $85 \%$ ethanol, then dried by vacuum centrifugation. rPDGF-BB in $0.25 \%$ HSA, $10 \mathrm{mM} \mathrm{NaOAc}, 0.15 \mathrm{M}$ $\mathrm{NaCl}$ was added to the dried pellets, tapped to ensure uniform distribution, then dried by vacuum centrifugation. Each sample received identical amounts of carrier solutions.

To measure rPDGF-BB release from ICBM in vitro, seven pellets were made as described above, without osteogenin. The dried pellets containing $54 \mu \mathrm{g}{ }^{125} \mathrm{I}-\mathrm{rPDGF}-\mathrm{BB}(29,000 \mathrm{cpm} / \mu \mathrm{g})$, chondroitin sulfate, and buffer salts were rehydrated with $0.5 \mathrm{ml} 0.25 \% \mathrm{HSA}$, then placed in an oscillating heating block at $37^{\circ} \mathrm{C}$. Radioactivity of $10-\mu \mathrm{l}$ aliquots was measured at designated times. Aliquots were frozen and subsequently assayed for bioactivity (mitogen assay) as described (11).

Surgical implantation. 144 male Long-Evans rats, 28-35 d old, were randomly assigned to treatment groups. 8-mm craniotomy defects were created in anesthetized rats as described previously (28). Implants were placed only into nonbleeding wounds. Soft tissue was closed with surgical staples. $28 \mathrm{~d}$ later, the rats were killed by $\mathrm{CO}_{2}$ asphyxiation. The fronto-occipital-parietal complex was removed and immersed in $70 \%$ ethanol.

Radiomorphometry. Specimens were radiographed with ultra-highcontrast mammography $\mathrm{x}$-ray film (X-Omat TL; Eastman Kodak Company, Rochester, NY) within $24 \mathrm{~h}$ of retrieval. Radiopacity was measured by computer-assisted image analysis $(28,36)$, and expressed as an area percentage of the $50.26-\mathrm{mm}^{2}$ defect.

Histomorphometry. Specimens were dehydrated with increasing concentrations of ethanol, then embedded in polymethylmethacrylate. Consecutive coronal sections, $4.5-5.0 \mu \mathrm{m}$ thick, were stained with von Kossa stain. The area of new bone calcium within defects was quantified by computer-assisted image analysis $(28,36)$.

Statistical analyses. Data were analyzed by graphics (Cricket Graph; Cricket Software, Malvern, PA) and statistical (Statview II; Abacus Concepts, Berkeley, CA ) computer software packages. We predicted that both OG and rPDGF-BB would have a positive, dose-related effect on bone regeneration within the defect site. Therefore, multiple regression analysis was performed on the data set. Fisher's protected least significant difference test was used to detect significant differences between the means of rPDGF-BB-treated and untreated groups within each dose of OG. Statistical significance was established at $P<0.05$.

\section{Results}

An initial dose-response study allowed determination of the effective dose range of partially purified OG. Fig. 1 shows the 28-d accumulation of radiopaque material within 8-mm craniotomy defects treated with $0,2,4,8,16,32,64,128$, and $256 \mu \mathrm{g}$ OG reconstituted with ICBM and chondroitin sulfate. Implantation of ICBM alone resulted in 15\% fill of the 8-mm defect with radiopaque material. Histological analysis identified this material as bone (not shown).

We investigated the effects of rPDGF-BB on near-optimal and suboptimal doses of $O G$. Computerized curve fitting of the dose-response data indicated the doses of OG required for $50 \%$ (half-maximal) and $90 \%$ of maximal response were $\sim 30$ and $\sim 150 \mu \mathrm{g}$, respectively. These doses and a $0-\mu \mathrm{g}$ OG control were chosen for combination with rPDGF-BB.

To estimate the release kinetics of rPDGF-BB from ICBM in vivo, the release of ${ }^{125} \mathrm{I}-\mathrm{rPDGF}-\mathrm{BB}$ from $25 \mathrm{mg}$ ICBM in vitro was measured (Fig. 2). The data confirm that rPDGF-BB has an affinity for ICBM; $<20 \%$ of the radiolabeled rPDGF$\mathrm{BB}$ was released into the supernatant within the first $1 \mathrm{~h}$, and $<50 \%$ was solubilized by $48 \mathrm{~h}$, when it approached plateau. Bioassay of the supernatant revealed that the soluble protein retained activity. We estimated that a dose of $200 \mu \mathrm{g}$ rPDGFBB reconstituted with ICBM would result in $40 \mu \mathrm{g}$ protein solubilized within the first $1 \mathrm{~h}$ after treatment. Likewise, a dose of $20 \mu \mathrm{g}$ rPDGF-BB reconstituted with ICBM would release 10 $\mu \mathrm{g}$ within the first $48 \mathrm{~h}$. We also chose $60 \mu \mathrm{g}$ as an intermediate dose. It was assumed that the presence of OG would not markedly alter the release kinetics of rPDGF-BB from ICBM.

To test the osteoinductivity of $O G$ and rPDGF-BB, 12 rats each were treated with 1 of 12 combinations of $O G$ and rPDGF-BB. Of the 144 rats treated, 11 died from anaesthesia or surgical complications within the first $4 \mathrm{~h}$ after surgery. Another rat was found dead the following morning. The remaining were ambulatory, alert, and responsive.

$11 \mathrm{~d}$ after surgery, a subcutaneous, cyst-like mass centered over the experimental defect was seen in 21 animals. This response had not been noted in previous experiments testing bone-inductive materials, including $O G$, and no masses were noted in animals treated with OG alone. The cyst-like masses varied in size and were scored on a four-point scale, 4 having a vertical elevation from the skull of $\sim 2 \mathrm{~cm}$ (Table I). The scorer was blinded to treatment groups. Multiple regression analysis showed that both rPDGF-BB and OG had significant dose effects on the size of the masses. However, the effect of $O G$ was significant only at the highest dose of rPDGF-BB, whereas

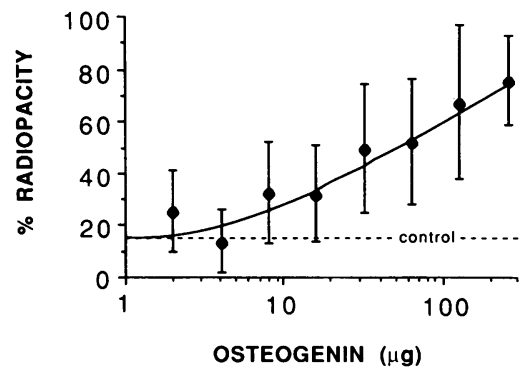

Figure 1. Radiopacity within 8-mm defect in response to increasing doses of OG. OG was reconstituted with ICBM as stated in Methods. Defect sites were retrieved after 28 d. Radiopacity is presented as a percentage of the total area of the

8-mm circle. Data represent mean and SD of 6-11 defects/dose. Regression analysis showed the dose effect to be significant $(P<0.05)$. 


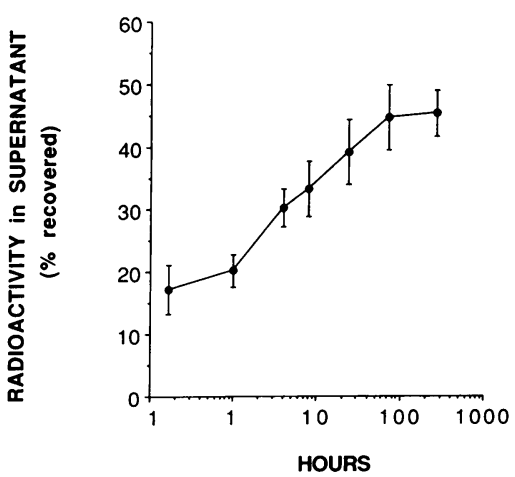

the effect of rPDGF-BB was significant at both doses of OG. The behavior of the affected rats did not deviate from normal.

One rat with a size 4 mass was killed to allow evaluation of the mass. Necropsy revealed a subcutaneous encapsulated mass attached to the calvarium at the defect margins (Fig. $3 \mathrm{~A}$ ). The tissue was turgid but elastic and was easily dissected from the overlying skin. Removal of the cranium and attached tissue mass revealed dura that appeared normal and cerebral cortices without edema, necrosis, or inflammatory exudate. No fluid leaked from the mass when it was necropsied.

Histological examination (Fig. $3 B$ ) revealed a core of proteinaceous fluid and erythrocyte ghosts with fibrin strands and occasional macrophages and neutrophils. The mass was encapsulated by fibrous tissue comprised of loose extracellular matrix admixed with macrophages and neutrophils. The ICBM had been displaced lateral and superior to the temporal crests of the parietal bones.

The masses resolved within the following 2 wk. At necropsy, however, experimental sites revealed fluid accumulation in several cases treated with $200 \mu \mathrm{g}$ rPDGF-BB. In two cases, clear yellow fluid had accumulated within the soft tissue superior to the defect site. In one case, sero-sanguineous fluid was observed supradurally, causing compression necrosis of a section of the cerebral cortex. Three additional sites treated with $200 \mu \mathrm{g}$ rPDGF-BB revealed focal necrosis of the cerebral cortex. Histologic examination confirmed compression and proliferation of the meninges. Another specimen had dilation of the right lateral ventricle, while in another there was evidence of hemorrhage into the lateral ventricle and into the subarachnoid space. There was a focal area of mineralization within one ventricle.

Radio- and histomorphometry (Fig. 4, $A$ and $B$ ) showed that $\mathrm{OG}$ had a positive dose effect on both percent radiopacity and regeneration of calcified tissue, whereas rPDGF-BB has a significant, negative dose effect only on percent radiopacity $(P$ $<0.05$ ). Comparisons of individual means indicated that all doses of rPDGF-BB inhibited OG-induced bone regeneration when the measured parameter was the percent radiopacity of the 8-mm defect (Fig. $4 A$ ). Measurements of new bone in coronal sections of defect sites indicated that at the higher dose of OG, only the 60- $\mu$ g dose of rPDGF-BB was inhibitory (Fig. 4 $B)$. The greatest effect was seen in the group treated with $30 \mu \mathrm{g}$ OG, where rPDGF-BB reduced by half the amount of radiopaque/calcified tissue regenerated in the defect.

\section{Histologic examination}

ICBM. Defects treated with ICBM alone formed an unremarkable scar with minimal new bone formation and a paucity of new marrow emanating from the host bone edges. Residual fragments of ICBM were surrounded by loose swirls of collagen fibers and fusiform-shaped fibroblasts (Fig. 5, $A-I$ and $A-2$ ).

$30 \mu g O G / I C B M$. Newly calcified woven bone extended from host margins and, in general, restored osseous contour to the craniotomy defects. The new bone was richly endowed in marrow containing typical cells, such as megakaryocytes, myeloid, and reticulocyte precursors. Robust connective tissue lining developed pericranially, whereas along the dura the connective tissue was more delicate. There were isolated areas of

Table I. Effect of Treatment on Size and Frequency of Soft Tissue Masses at 11 d Postimplantation

\begin{tabular}{|c|c|c|c|c|c|c|c|c|c|}
\hline \multirow{2}{*}{\multicolumn{2}{|c|}{ Treatments }} & \multicolumn{5}{|c|}{ Number } & & & \multirow{3}{*}{$\begin{array}{l}\text { Mean size } \\
\text { (SD) }\end{array}$} \\
\hline & & \multirow[b]{2}{*}{ Total } & \multicolumn{4}{|c|}{ According to size of masses } & \multicolumn{2}{|c|}{ Frequency } & \\
\hline OG & PDGF & & 1 & 2 & 3 & 4 & Total & Percent & \\
\hline$\mu g$ & $\mu g$ & & & & & & & & \\
\hline 0 & 0 & 11 & 0 & 0 & 0 & 0 & 0 & 0 & $0(0)$ \\
\hline 0 & 20 & 11 & 0 & 0 & 0 & 0 & 0 & 0 & $0(0)$ \\
\hline 0 & 60 & 11 & 0 & 0 & 0 & 0 & 0 & 0 & $0(0)$ \\
\hline 0 & 200 & 12 & 1 & 0 & 0 & 0 & 1 & 8 & $0.08(0.29)$ \\
\hline 30 & 0 & 11 & 0 & 0 & 0 & 0 & 0 & 0 & $0(0)$ \\
\hline 30 & 20 & 10 & 0 & 0 & 0 & 0 & 0 & 0 & $0(0)$ \\
\hline 30 & 60 & 11 & 0 & 1 & 0 & 0 & 1 & 9 & $0.18(0.60)$ \\
\hline 30 & 200 & 11 & 5 & 2 & 0 & 0 & 7 & 64 & $0.82(0.75)^{* \ddagger}$ \\
\hline 150 & 0 & 11 & 0 & 0 & 0 & 0 & 0 & 0 & $0(0)$ \\
\hline 150 & 20 & 11 & 0 & 1 & 0 & 0 & 1 & 9 & $0.18(0.60)$ \\
\hline 150 & 60 & 10 & 2 & 0 & 0 & 0 & 2 & 20 & $0.20(0.42)$ \\
\hline 150 & 200 & 12 & 2 & 3 & 2 & 2 & 9 & 75 & $1.83(1.47)^{* \ddagger}$ \\
\hline
\end{tabular}

Mean size was compared with appropriate controls using Fisher's protected least significance difference test. * Significantly different from $0 \mu \mathrm{g}$ OG control at same dose of PDGF $(P<0.05)$. ${ }^{\ddagger}$ Significantly different from $0 \mu \mathrm{g}$ PDGF control at same dose of $\mathrm{OG}(P<0.05)$. 

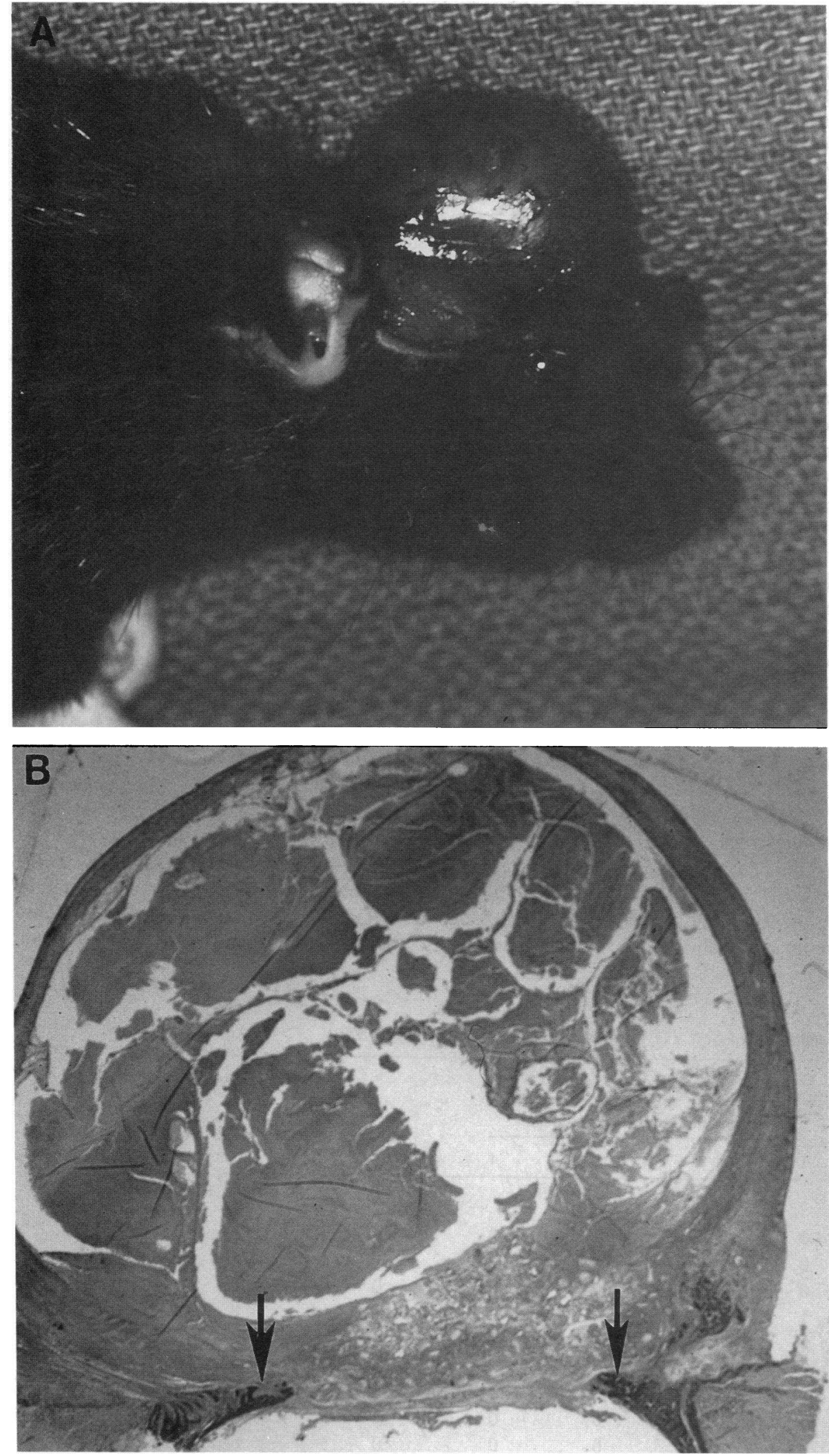

Figure 3. (A) At necropsy, the encapsulated mass (hematocoel) $11 \mathrm{~d}$ after treatment. $(B)$ Section of hematocoel showing core of proteinaceous fluid and erythrocyte ghosts and fibrin strands, occasional macrophages, neutrophils, and fibrous tissue composed of loose extracellular matrix admixed with macrophages and neutrophils. ICBM displaced lateral and superior to the temporal crests of the parietal bones. Parietal bone margin (arrows) $(\times 2$; Hematoxylin and Eosin stain) fusiform-shaped, streaming fibroblasts. Occasional remnants of ICBM were seen superior to the new bone. Vascularity was rich and consisted of many venules, arterioles, and capillaries.

$150 \mu g O G / I C B M$. In general, this treatment group looked similar to the $30-\mu \mathrm{g} \mathrm{OG}$ treatment group. There was bony heal- ing across the critical-sized defect ( Fig. $5 B$ ), infrequent islands of fibrocartilage, and occasional foci of cartilage. In addition, woven bone appeared to predominate over lamellar bone when compared with the $30 \mu \mathrm{g}$ OG-treated group. Healing tissue consisted of rich inosteoblasts, each with a large nucleus and 

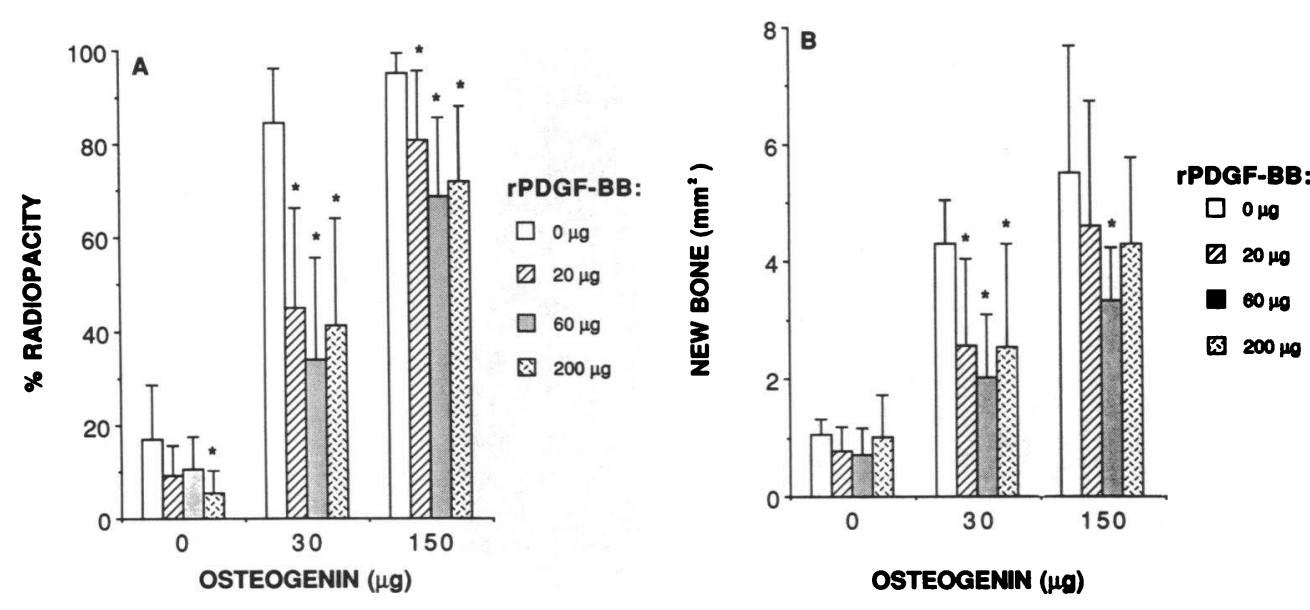

Figure 4. Effect of $O G$ and rPDGF-BB on bone regeneration within 8-mm craniotomy defects. $(A)$ Radiopaque material within defect site reported as a percentage of the defect area. $(B)$ Area of new calcified tissue within coronal midsection of defect site as measured by histomorphometry. Data represent the mean and SD of 10-12 defects per treatment group. OG had a positive dose effect on both percent radiopacity and regeneration of calcified tissue, whereas rPDGF-BB had a significant, negative dose effect only on percent radiopacity. *significant difference between rPDGF-BBtreated and untreated groups within each dose of OG $(P<$ $0.05)$.

ample cytoplasm. Fibroblasts were interspersed in lush collagen bundles.

$20 \mu g r P D G F-B B / I C B M$. There were numerous remnants of ICBM with a paucity of new bone activity along pericranial host bone margins (Fig. $5 C$ ). Osteoblasts were few in number with rare osteoclasts. Fibrocartilage was observed subjacent to the pericranial connective tissue lining in two cases. There was nothing unusual about either the cellular or stromal elements of the tissue.

$60 \mu g r P D G F-B B / I C B M$. Observations for this treatment group were essentially identical to those described for $20 \mu \mathrm{g}$ PDGF. Occasionally, remnants of a supradural hematoma were evident in a few specimens with fibrin strands and minimal number of macrophages. The granulation tissue consisted of numerous swirls of collagenous fibers, fibroblasts with robust nuclei, and rich neovascularization. Again, there was either little or no new bone. Osteoblastic activity was confined to some pericranial aspects of the host bone; it was not associated with the ICBM particles containing PDGF. Isolated islands of fibrocartilage were apparent.

$200 \mu g r P D G F-B B / I C B M$. There were numerous remnants of ICBM throughout the defect (Fig. $5 \mathrm{D}$ ), wrapped in a loose web of connective tissue with a moderate number of fibroblasts. New bone formation from host margins was sparse. One specimen in this group displayed an isolated area of fibrocartilage mid-defect along the pericranial aspect. Neovascularization was rich, with numerous venules, arterioles, and capillaries.

$30 \mu g O G / 20 \mu g r P D G F-B B / I C B M$. A wispy connective tissue stroma surrounded nonreactive ICBM particles with a typical component of capillaries, venules, and arterioles. There was some new bone formation superior to and emanating from the bone wound margins. Less bone developed in response to this treatment than that observed in response to $30 \mu \mathrm{g}$ OG. Occasional osseous bridging of the craniotomy occurred with restoration of form. Marrow, stromal, and cellular elements were normal in appearance.

$30 \mu g O G / 60 \mu g r P D G F-B B / I C B M$. There were degenerating ICBM particles throughout the craniotomy wound surrounded by loose granulation tissue and plentiful, ellipsoi- dal to slightly rounded fibroblasts. Along the dura and pericranial aspects the connective tissue capsule appeared thicker and more condensed than in response to $30 \mu \mathrm{g} \mathrm{OG}$ alone. The connective tissue along the superior aspect of the surgical site appeared to have more arterioles than other treatment groups (Fig. $5 E$ ). Furthermore, new bone formation seemed more subdued than that observed in other $O G$ treatments.

$30 \mu g O G / 200 \mu g r P D G F-B B / I C B M$. Dense connective bridging spanned the $8-\mathrm{mm}$ craniotomy wounds displaying scant new bone formation associated with ICBM. Bone regeneration occurred predominantly at host wound edges in a centripetal direction. There was more woven bone pericranially than along the dura, indicating increased activity in this zone in contrast to other areas of the healing wound. Several specimens revealed a dense connective capsule surrounding a resolving hematoma.

$150 \mu g O G / 20 \mu g \mathrm{rPDGF}-\mathrm{BB} / I C B M$. There were bone bridges across the craniotomies consisting of condensing woven and lamellar bone, rich marrow, active osteoblasts, and subjacent osteoid (Fig. $5 F-1$ ). Numerous capillaries, venules, and arterioles were seen along the dura; a slightly greater amount was observed pericranially. Residual fragments of ICBM were amalgamated within the newly forming bone with nidi of independent zones of new bone (Fig. $5 F-2$ ). Osteoclasts were occasionally observed lining isolated zones of new bone. Collagenous whirls were infrequent, as compared with craniotomies treated with a lower dose of OG. There were some foci (mid-superior defect) of fibro-osseous tissue with ellipsoid- and fusiform-shaped fibroblasts and chondrogenic cells.

$150 \mu g$ OG $/ 60 \mu g \mathrm{rPDGF}-\mathrm{BB} / \mathrm{ICBM}$ and $150 \mu \mathrm{g} O G / 200$ $\mu g r P D G F-B B / I C B M$. Histologically, specimens from these treatment groups appeared similar to the previous group. Cells and stroma were normal in appearance and without evidence of the hematocoel that was observed at $11 \mathrm{~d}$.

\section{Discussion}

Stimulation of bone regeneration within CSDs treated with bone-inductive factors were confirmed and extended in the present experiments; $150 \mu \mathrm{g}$ of $\mathrm{OG}$ reconstituted with $25 \mathrm{mg}$ 

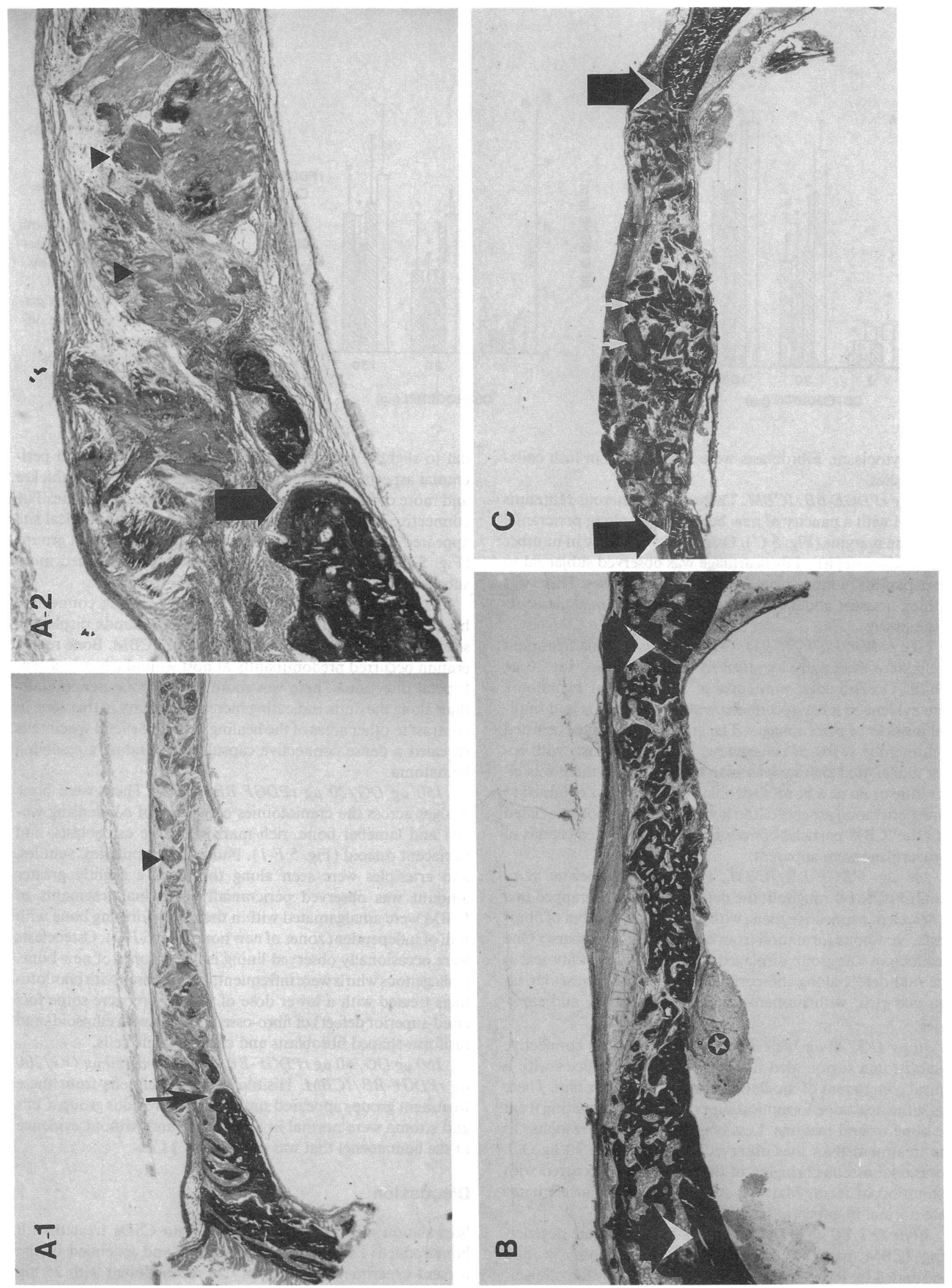

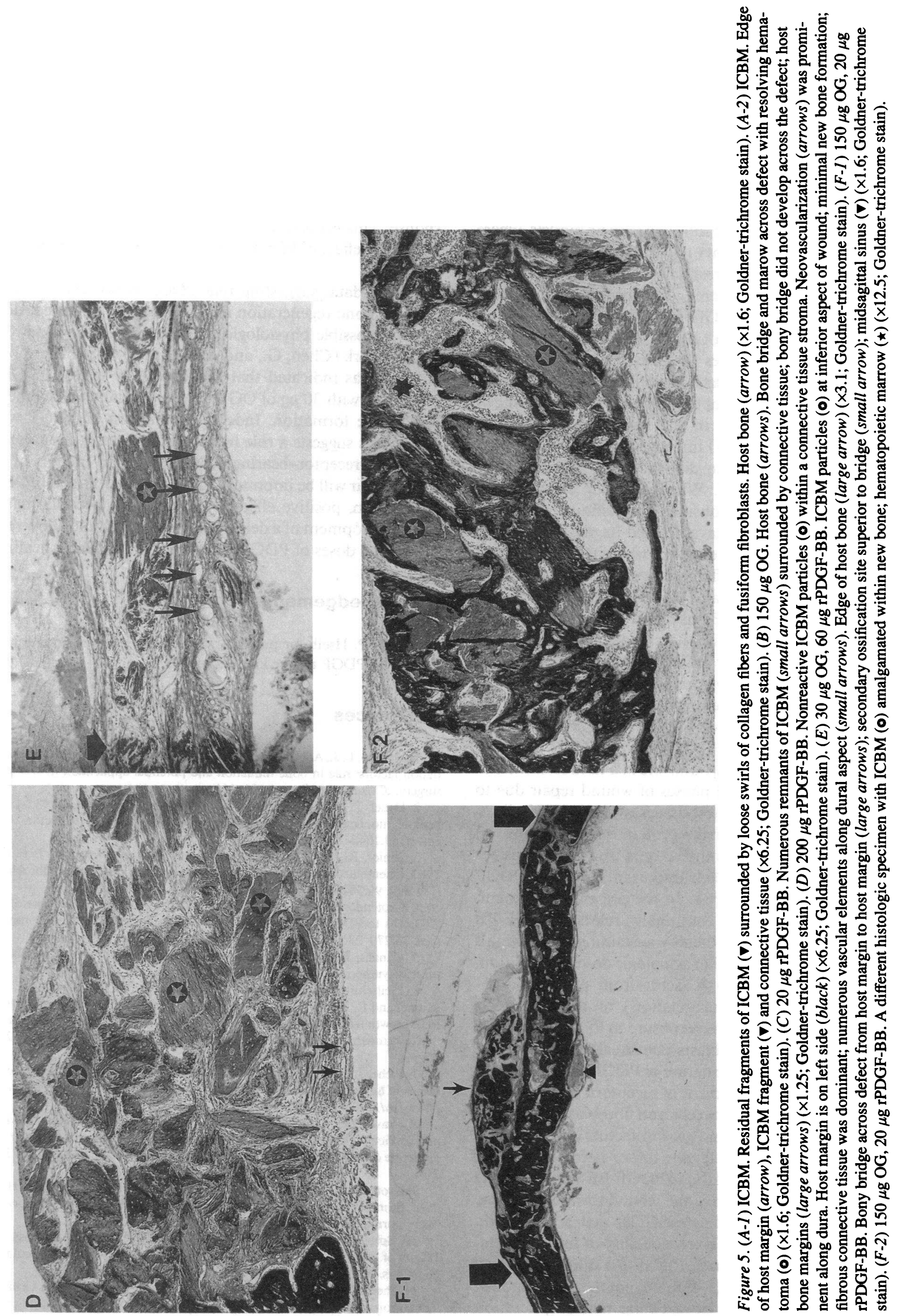
ICBM resulted in nearly $100 \%$ regeneration of CSDs within 28 d. Whereas we expected rPDGF-BB to enhance OG-induced bone regeneration, we actually found decreased osteogenesis. This inhibition was probably secondary to a soft tissue repair phenotype induced within the wound by rPDGF-BB. The data suggest the detrimental effect of rPDGF-BB on bone formation might be overcome with a higher dose of OG.

The soft tissue response observed in some defects treated with rPDGF-BB most resembles that of a sustained inflammatory reaction. The inflammation seemed to have resolved by the time the animals were killed (day 28). These results are consistent with observations of soft tissue repair models, where exaggerated repair was transient (17). Parenchymal changes to brains probably occurred in response to pressure exerted by accumulated fluid, whereas meningeal proliferation may indicate a mitogenic effect of rPDGF-BB on these tissues.

It is possible that the doses of rPDGF-BB used in this experiment were too high. The doses were based on reported effective ranges of PDGF. Pierce and coworkers $(15,16)$ showed that a topical dose of $5 \mu \mathrm{g}$ was optimal in stimulating healing of a $28-\mathrm{mm}^{2}$ excisional wound in the ear dermis of rabbits, whereas Greenhalgh et al. (14) improved repair of $225-\mathrm{mm}^{2}$, full-thickness skin excisions in diabetic mice with five daily applications of $10 \mu \mathrm{g}$ PDGF. When choosing experimental doses of rPDGF-BB, we also considered the affinity of rPDGFBB for ICBM, which might have delayed its activity in vivo. PDGF probably exerts it greatest effect when its concentration is highest, during the initial inflammatory phase when platelets enter the wound and degranulate. We wanted to ensure that an effective amount of rPDGF-BB was released from the ICBM by this time. The slow release in vitro necessitated the use of a high dose of rPDGF-BB to ensure release of an optimal dose within the critical time frame. However, release kinetics in vivo might be hastened by the action of extracellular enzymes, acidification of the wound area, and degradation of ICBM.

The sustained release of rPDGF-BB from ICBM probably maintained the protein at the wound site in a fashion similar to what occurs during the initial phases of wound repair due to platelet influx. Its half-life in most tissues is $<4 \mathrm{~h}$ (37), indicating the body's ability to clear the protein quickly. However, macrophages, fibroblasts, endothelial cells, and perhaps osteoblasts and chondroblasts are likely important sources of PDGF throughout wound healing (38). In the present experiment, degradation of ICBM could continually release rPDGF-BB into the extracellular milieu, thereby sustaining the protein's presence at the wound site at pharmacologic doses. In addition, ICBM may protect the growth factor from proteolytic enzymes. Internalization (downregulation) of receptors (39) may protect local cells from overexposure to PDGF, but naive inflammatory cells and fibroblasts continually drawn to the wound bed by the chemotactic nature of PDGF would presumably have the normal complement of receptors. In addition to PDGF, activated inflammatory cells and fibroblasts synthesize a large number of cytokines and growth factors (e.g., interleukin-1, basic fibroblast growth factor, tumor necrosis factor- $\alpha$, interferons, etc.) that can sustain a rPDGF-BB-induced acute inflammatory reaction and drive the wound toward a chronic inflammatory state, leading to extracellular matrix deposition and a large soft tissue mass. The reversibility of the phenomenon suggests that once the initial rPDGF-BB stimulus is gone, the inflammatory reaction is self-limited, but the cells responsible for soft tissue repair and extracellular matrix generation (fibroblasts) have repaired the bony defect with nonbone matrix. Consequently, overabundance of the typical cells and cellular products required in soft tissue repair likely triggered the development of soft tissue masses and drove the repair process away from a hard tissue phenotype.

The precise mode of action of osteogenin and related bone morphogenetic protein(s) is not known. However, it is likely that the responding mesenchymal stem cells have the potential to proceed on at least two different lineages: soft tissue or hard skeletal tissue. In view of this, there is a delicate balance that is modified by PDGF-BB toward a predominantly soft tissue response. This is borne out by the observed lack of pronounced inhibitory effect of PDGF-BB when higher doses of osteogenin were used.

These data, suggesting that PDGF is not effective in enhancing bone regeneration at the dosage levels tested, do not refute a possible physiological role for PDGF in bone repair. Recent work (Chen, G., and A. H. Reddi, unpublished observations) has indicated that low doses (50 ng) of PDGF-BB combined with $30 \mu \mathrm{g}$ of OG act synergistically to elicit heterotopic bone formation. Indeed, localization of PDGF within osteoblasts suggests a role in bone homeostasis. Identification of PDGF receptor-bearing cells $(40,41)$ in models of hard tissue repair will be important in determining targets of PDGF. In addition, positive effects of PDGF may become manifest after development of a delivery system that would allow release of optimal doses of PDGF at optimal times during healing.

\section{Acknowledgements}

We thank P. Hseih for purified rPDGF-BB and S.-Z. Song for assistance with PDGF release kinetics studies.

\section{References}

1. Marden, L. J., A. H. Reddi, and J. O. Hollinger. 1990. Growth and differentiation factors: role in bone induction and potential application in craniofacial surgery. J. Craniofac. Surg. 1:154-161.

2. Deuel, T. F., R. M. Senior, J. S. Huang, and G. L. Griffen. 1982. Chemotaxis of monocytes and neutrophils to platelet-derived growth factor. J. Clin. Invest. 69:1046-1049.

3. Senior, R. M., G. L. Griffins, J. S. Huang, D. A. Walz, and T. F. Deuel. 1983. Chemotactic activity of platelet alpha granule proteins for fibroblasts. J. Cell Biol. 96:382-385.

4. Grotendorst, G. R. 1984. Alteration of the chemotactic response of NIH/ 3 T3 cells to PDGF by growth factors, transformation, and tumor promoters. Cell. 36:279-285.

5. Canalis, E. 1981. Effect of platelet-derived growth factor on DNA and protein synthesis in cultured rat calvaria. Metabolism. 30:970-975.

6. Pfeilschifter, J., M. Oechsner, A. Naumann, R. G. K. Gronwald, H. W. Minne, and R. Ziegler. 1990. Stimulation of bone matrix apposition in vitro by local growth factors: a comparison between insulin-like growth factor I, plateletderived growth factor, and transforming growth factor $\beta$. Endocrinology. 127:6975.

7. Piché, J. E., and D. T. Graves. 1989. Study of the growth factor requirements of human bone-derived cells: a comparison with human fibroblasts. Bone (Elmsford). 10:131-138.

8. Graves, D. T., A. Valentin-Opran, R. Delgado, A. J. Valente, G. Mundy, and J. Piché. 1989. The potential role of platelet-derived growth factor as an autocrine or paracrine factor for human bone cells. Connect. Tissue Res. 23:209218.

9. Grotendorst, G. R., G. R. Martin, D. Pencev, J. Sodek, and A. K. Harvey. 1985. Stimulation of granulation tissue formation by platelet-derived growth factor in normal and diabetic rats. J. Clin. Invest. 76:2323-2329.

10. Sprugel, K. H., J. M. McPherson, A. W. Clowes, and R. Ross. 1987. Effects of growth factors in vivo. 1. Cell ingrowth into porous subcutaneous chambers. Am. J. Pathol. 129:601-613.

11. Pierce, G. F., T. A. Mustoe, R. M. Senior, J. Reed, G. L. Griffen, A. Thomason, and T. F. Deuel. 1988. In vivo incisional wound healing augmented 
by platelet-derived growth factor and recombinant c-sis gene homodimeric proteins. J. Exp. Med. 167:974-987.

12. Pierce, G. F., T. A. Mustoe, J. Lingelbach, V. R. Marakowski, P. Gramates, and T. F. Deuel. 1989. Transforming growth factor $\beta$ reverses the glucocorticoid-induced wound-healing deficit in rats: possible regulation in macrophages by platelet-derived growth factor. Proc. Natl. Acad. Sci. USA. 86:22292233.

13. Pierce, G. F., T. A. Mustoe, J. Lingelbach, V. Masakowski, and G. L. Griffin. 1989. Platelet-derived growth factor and transforming growth factor- $\beta$ enhance tissue repair activities by unique mechanisms. J. Cell Biol. 109:429-440.

14. Greenhalgh, D. G., K. H. Sprugel, M. J. Murray, and R. Ross. 1990. PDGF and FGF stimulate wound healing in the genetically diabetic mouse. Am. J. Pathol. 136:1235-1246.

15. Mustoe, T. A., G. F. Pierce, C. Morishima, and T. F. Deuel. 1991. Growth factor-induced acceleration of tissue repair through direct and inductive activities in a rabbit dermal ulcer model. J. Clin. Invest. 87:694-703.

16. Pierce, G. F., J. Vande Berg, R. Rudolph, J. Tarpley, and T. A. Mustoe. 1991. Platelet-derived growth factor-BB and transforming growth factor betal selectively modulate glycosaminoglycans, collagen, and myofibroblasts in excisional wounds. Am. J. Pathol. 138:629-646.

17. Pierce, G. F., J. E. Tarpley, D. Yanagihara, T. A. Mustoe, G. M. Fox, and A. Thomason. 1992. Platelet-derived growth factor (BB homodimer), transforming growth factor- $\beta 1$, and basic fibroblast growth factor in dermal wound healing. Am. J. Pathol. 140:1375-1388.

18. Howes, R., J. M. Bowness, G. R. Grotendorst, G. R. Martin, and A. H. Reddi. 1988. Platelet-derived growth factor enhances demineralized bone matrixinduced cartilage and bone formation. Calcif. Tissue Int. 42:34-38.

19. McGill, J. J., B. S. Strates, and M. H. McGuire. 1991. Stimulation of osteogenesis by PDGF and TGF $\beta$ adsorbed on microcrystalline hydroxyapatite. J. Bone Miner. Res. 6:S210.

20. Noda, M., J. J. Camilliere, and G. A. Rodan. 1988. Transforming growth factor type $\beta$ promotes bone formation in vivo. J. Cell Biol. 107:48a. (Abstr.)

21. Noda, M., and J. J. Camilliere. 1989. In vivo stimulation of bone formation by transforming growth factor- $\beta$. Endocrinology. 124:2991-2994.

22. Russell, S. M., and E. M. Spencer. 1985. Local injections of human or rat growth hormone or of purified human somatomedin-C stimulate unilateral tibial epiphyseal growth in hypophysectomized rats. Endocrinology. 116:2563-2567.

23. Joyce, M. E., A. B. Roberts, M. B. Sporn, and M. E. Bolander. 1990. Transforming growth factor- $\beta$ and the initiation of chondrogenesis and osteogenesis in the rat femur. J. Cell Biol. 110:2195-2207.

24. Schlechter, N. L., S. M. Russell, E. M. Spencer, and C. S. Nicoll. 1986. Evidence suggesting that the direct growth-promoting effect of growth hormone on cartilage in vivo is mediated by local production of somatomedin. Proc. Natl. Acad. Sci. USA. 83:7932-7934.

25. Eppley, B. L., M. Doucet, D. T. Connolly, and J. Feder. 1988. Enhancement of angiogenesis by bFGF in mandibular bone graft healing in the rabbit. $J$. Oral Maxillofac. Surg. 46:391-398.
26. Sampath, T. K., and A. H. Reddi. 1981. Dissociative extraction and reconstitution of extracellular matrix components involved in local bone differentiation. Proc. Natl. Acad. Sci. USA. 78:7599-7603.

27. Sampath, T. K., and A. H. Reddi. 1983. Homology of bone-inductive proteins from human, monkey, bovine, and rat extracellular matrix. Proc. Natl. Acad. Sci. USA. 80:6591-6595.

28. Mark, D. E., J. O. Hollinger, C. Hastings, S. Ma, G. Chen, L. J. Marden, and A. H. Reddi. 1990. Repair of calvarial nonunions by osteogenin, a bone-in ductive protein. Plast. Reconstr. Surg. 86:623-630.

29. Smith, J. C., J. P. Singh, J. S. Lillquist, D. S. Goon, and C. D. Stiles. 1982 Growth factors adherent to cell substrate are mitogenically active in situ. Nature (Lond.). 296:154-156.

30. Hollinger, J. O., and J. C. Kleinschmidt. 1990. The critical size defect as an experimental model to test bone repair materials. J. Craniofac. Surg. 1:60-68.

31. Schmitz, J. P., Z. Schwartz, J. O. Hollinger, and B. D. Boyan. 1990 Characterization of rat calvarial nonunion defects. Acta Anat. 138:185-192.

32. Vukicevic, S., F. P. Luyten, and A. H. Reddi. 1989. Stimulation of the expression of osteogenic and chondrogenic phenotypes in vitro by osteogenin. Proc. Natl. Acad. Sci. USA. 86:8793-8797.

33. Vukicevic, S., F. P. Luyten, and A. H. Reddi. 1990. Osteogenin inhibits proliferation and stimulates differentiation in mouse osteoblast-like cells (MC3T3-E1). Biochem. Biophys. Res. Commun. 166:750-756.

34. Luyten, F. P., N. S. Cunningham, S. Ma, N. Muthukumaran, R. G. Hammonds, W. B. Nevins, W. I. Wood, and A. H. Reddi. 1989. Purification and partial amino acid sequence of osteogenin, a protein initiating bone differentiation. J. Biol. Chem. 264:13377-13380.

35. Muthukumaran, N., S. Ma, and A. H. Reddi. 1988. Dose-dependence of and threshold for optimal bone induction by collagenous bone matrix and osteogenin-enriched fraction. Collagen Relat. Res. 8:433-441.

36. Hollinger, J. O., J. P. Schmitz, D. E. Mark, and A. E. Seyfer. 1990. Osseous wound healing with xenogeneic bone implants with a biodegradable carrier. Surgery (St. Louis). 107:50-54.

37. Cohen, A. M., C. Sodenberg, and A. Thomason. 1990. Plasma clearance and tissue distribution of recombinant human platelet-derived growth factor (Bchain homodimer) in rats. J. Surg. Res. 49:447-452.

38. Pierce, G. F., T. A. Mustoe, B. W. Altrock, T. F. Deuel, and A. Thomason. 1991. Role of platelet-derived growth factor in wound healing. J. Cell. Biochem. 45:319-326.

39. Williams, L. T. 1989. Signal transduction by the platelet-derived growth factor receptor. Science (Wash. DC). 243:1564-1570.

40. Centrella, M., T. L. McCarthy, W. F. Kusmik, and E. Canalis. 1991. Relative binding and biochemical effects of heterodimeric and homodimeric isoforms of platelet-derived growth factor in osteoblast-enriched cultures from fetal rat bone. J. Cell. Physiol. 147:420-426.

41. Centrella, M., T. L. McCarthy, W. F. Kusmik, and E. Canalis. 1992. Isoform-specific regulation of platelet-derived growth factor activity and binding in osteoblast-enriched cultures from fetal rat bone. J. Clin. Invest. 89:1076-1084. 